\title{
SketchSPORE: A Sketch Based Domain Separation and Recognition System for Interactive Interfaces
}

\author{
Danilo Avola ${ }^{1}$, Luigi Cinque ${ }^{2}$, and Giuseppe Placidi ${ }^{1}$ \\ 1 Department of Life, Health and Environmental Sciences, University of L'Aquila \\ Via Vetoio Coppito 2, 67100, L'Aquila, Italy \\ \{danilo.avola, giuseppe.placidi\}@univaq.it \\ http://www . univaq. it/en/section . php?id=262 \\ 2 Department of Computer Science, Sapienza University \\ Via Salaria 113, 00198, Rome, Italy \\ cinque@di.uniroma1.it \\ http://w3.uniroma1.it/dipinfo/english/index.asp
}

\begin{abstract}
Multimodal interfaces are used to interact with devices and automata using different channels of communication. In this context, the sketch modality plays a key role since it allows users to convey concepts and/or commands using freehand drawing (graphical domain) and/or handwriting (textual domain). The acquisition of the sketch modality can be performed using touch (e.g., touchscreen) or touchless (e.g., RGB$\mathrm{D}$ camera) tools supporting the development of versatile and powerful interactive interfaces. Domain separation and sketch recognition are two fundamental issues of these interfaces. This paper presents SketchSPORE a novel framework designed both to automatically distinguish graphical from textual elements within the same sketch and to recognize freehand drawing as well as handwriting. The recognition processes support both on-line and off-line modes, moreover their processing can be suitably stored within an XML file to provide a means to maintain the compatibility between the framework and service and/or application targets. Extensive experiments showing the effectiveness of the proposed method are reported and discussed.
\end{abstract}

Keywords: multimodal interfaces, sketch recognition, freehand drawing, handwriting, graphical domain, textual domain, SketchML.

\section{Introduction}

Multimodal interfaces allow users to interact with devices (e.g., tablets, smartphones, game consoles) using multiple modalities (e.g., sketch, gesture, speech) according to application requirements, environmental characteristics (e.g., indoor, outdoor) or device-dependent features (e.g., screen size, computational capacity). More recently, some human-oriented modalities (e.g., gestures) have been widely used to interact with advanced systems (e.g., Computer Assisted 
Rehabilitation ENvironment, CAREN 11]) or automata (e.g., androids) reaching new frontiers of the human-computer interaction (HCI). In this context, the sketch modality is of primary importance since it allows users to convey concepts and/or commands using freehand drawing (e.g., graphical symbols) and/or handwriting (e.g., sentences). In addition, the recognition algorithms used to support this modality can be adapted to develop gesture-based interfaces. In fact, the acquisition of any body, hand or finger gesture by touch (e.g., body suites, haptic gloves) or touchless (e.g., RGB, ToF or RGB-D cameras) tools can be always treated like a set of skeletonized freehand drawings whose recognition supports the pose estimation and tracking of the related gesture [37]. The process of distinguishing freehand drawing from handwriting elements within the same sketch, as well as the recognition of these elements, are two fundamental issues of the sketch-based interfaces since many application contexts have both types of elements. Moreover, unlike the handwriting, the freehand drawing recognition is still suffering in terms of multi-domain and ambiguity issues. The first highlights the ability of a system in defining and recognizing any set of graphical symbols (hereinafter: library), the last underlines the difficulties that occur during the recognition of two similar (e.g., circle vs ellipse) and/or equivocal (e.g., infinity vs two near circles) symbols.

This papers presents SketchSPORE (Sketch SeParatiOn and REcognition) a novel framework designed both to automatically distinguish graphical from textual elements within the same sketch and to recognize freehand drawing as well as handwriting. The framework is composed by three main algorithms. The first detects how many objects have been sketched by users and their related domain. In this context, an object is a set (one or more) of strokes. The second and the third algorithms recognize the freehand drawing and the handwriting, respectively. In particular, the freehand drawing process has been designed to support the multi-domain issue, and an ambiguity solving method has been also implemented. This paper is focused on the first two algorithms for which novel approaches have been implemented. The last is referred to an ordinary method. Domain separation and sketch recognition algorithms support both on-line and off-line modes, moreover the outputs related to these algorithms are suitably stored within an eXtensible Markup Language (XML) file to provide a standard communication channel between the framework and service and/or application targets. The paper is based on advancements of some previous experiences found in literature about textual/graphical domain separation [5] and sketch-based interfaces [4]. Extensive experiments showing the recognition processes on a wide set of sketches are reported and discussed.

This paper is structured as follows. Section 2 introduces some remarkable works supporting domain separation and multi-domain sketch recognition fields. Section 3 presents a complete overview of the SketchSPORE framework detailing the domain separation and the graphical recognition algorithms; textual recognition algorithm is also referenced. Section 4 summarizes experimental results on a wide set of sketches composed by graphical and textual (uppercase and lowercase letters) elements. Finally, Section 5 concludes the paper. 


\section{Related Work}

In literature there are few works discussing the multi-domain graphical recognition, and still less regarding the domain separation. Moreover, there is not a framework based on experimental results regarding both processes (and that also includes the textual recognition) supporting the on-line and off-line modes as well as an ambiguity resolution approach. Despite this, several interesting works have supported our technical and theoretical choices. Regarding the multi-domain sketch recognition the works in 13]12 suggest a set of geometrical features and a fuzzy logic approach, combined with an extensible set of heuristics, to classify elementary geometric shapes. In our approach, we focused on a general recognition engine to identify any kind of shape, while maintaining recognition independent from size, rotation or style of the drawn shapes like the mentioned works. Other main works are shown in [12] where the authors present a multi-domain sketch recognition engine capable of recognizing diagrammatic freehand drawings. Their system can be applied to a variety of domains by providing related structural descriptions, moreover their method does not require training data or additional programming. Finally, robustness and ambiguity resolution are achieved through the use of a context. Different aspects of the mentioned papers (i.e., description language and selection of the interpretation) have contributed in developing some functional characteristics of the proposed algorithms. The works in [14|15] present a language to describe how sketched diagrams in a domain are drawn, displayed, and edited. Their language consists of predefined shapes, constraints, editing behaviors, and display methods, as well as a syntax for specifying a domain description sketch grammar and extending the language, ensuring that shapes and shape groups from many domains can be described. Another complete and versatile language is reported in [6] where the authors use it in storing shapes and matching processes.

Regarding the separation domain an interesting work is [8], where a system to distinguish between shape and text strokes is presented. The authors have found the entropy rate to be an accurate criterion of classification. We have applied similar measures to detect how many objects have been sketched by users, afterwards we have developed other measures to classify (graphical or textual) each object. Another remarkable work is described in [10] where the authors introduce a set of ink features combined with data mining techniques to build accurate text-shape dividers. A careful analysis of the dividers presented within the paper have confirmed our previous impressions related to some features and approaches. A meaningful work is detailed in [9] where machine learning techniques are used. In particular, the authors use the characteristics of the strokes, the information provided by the gaps between the strokes, and the temporal characteristics of the stroke sequence to define a probabilistic classifier able to lead the classification process. A different work is reported in [17, where the authors present a survey regarding the on-line and off-line handwriting recognition. The works cited within the survey as well as the dynamic measures adopted to characterize the stroke properties have influenced our approach in developing the set of features used in domain separation and sketch recognition issues. 


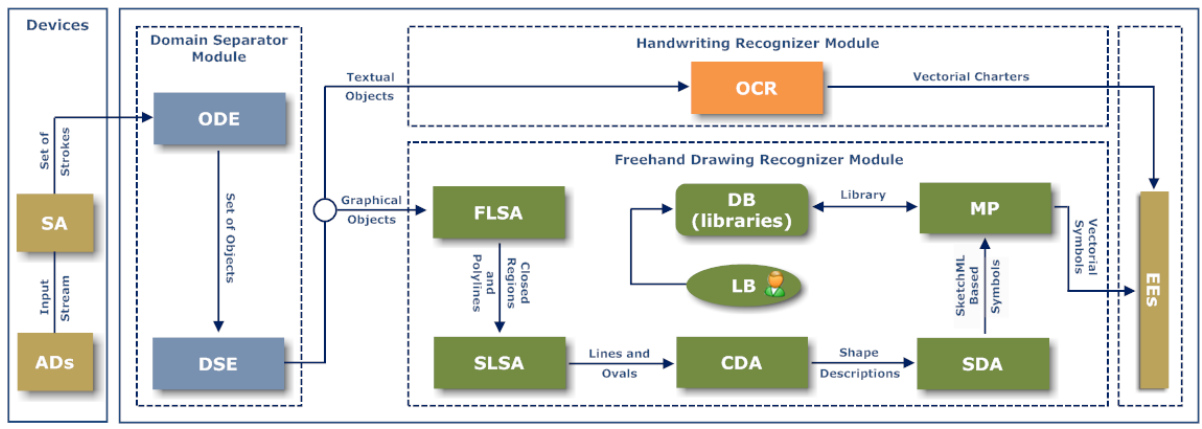

Fig. 1. SketchSPORE Architecture

\section{SketchSPORE Architecture}

As shown in Fig. 1, the framework is composed by three main modules: Domain Separator, Handwriting Recognizer, Freehand Drawing Recognizer. The first is responsible for determining and classifying each object within textual or graphical class; the second and third recognize handwriting and freehand drawing, respectively. In particular, the second module recognizes the strokes associated to each textual object and replaces them with a set of roman alphabet and/or numeral characters; while the third module adopts a previously defined library to replace the strokes associated to each graphical object with a specific vectorial symbol. Before detailing the modules of the architecture we introduce three processes that define the contexts of use of the framework: Acquisition Devices (ADs), Skeletonized Algorithm (SA), and External Environments (EEs). The first highlights that the framework has been designed to acquire input from any kind of device (e.g., tablets, touchscreen, RGB-D camera); the second points out that any input stream has to be preprocessed to provide a stroke based data structure containing pixel based information including ( $\mathrm{x}, \mathrm{y})$ coordinates and related acquisition time; the third shows that the result of the user interaction (i.e., recognized symbols and/or characters) can be used to convey concepts and/or commands useful to interact with devices and automata. The framework can work in on-line and off-line mode. In the first case the three processes run whenever a user ends drawing a stroke, in the second case they run only when a user provides a command on the framework GUI.

\subsection{Domain Separator Module}

This module is composed by two processes: Object Detection Engine (ODE), and Domain Separation Engine (DSE). The first detects how many and which objects have been sketched by users. The second classifies them within textual or graphical classes. The ODE checks temporal (i.e., sequential) and spatial (i.e., interception and closeness) relationships among strokes to provide one or more 

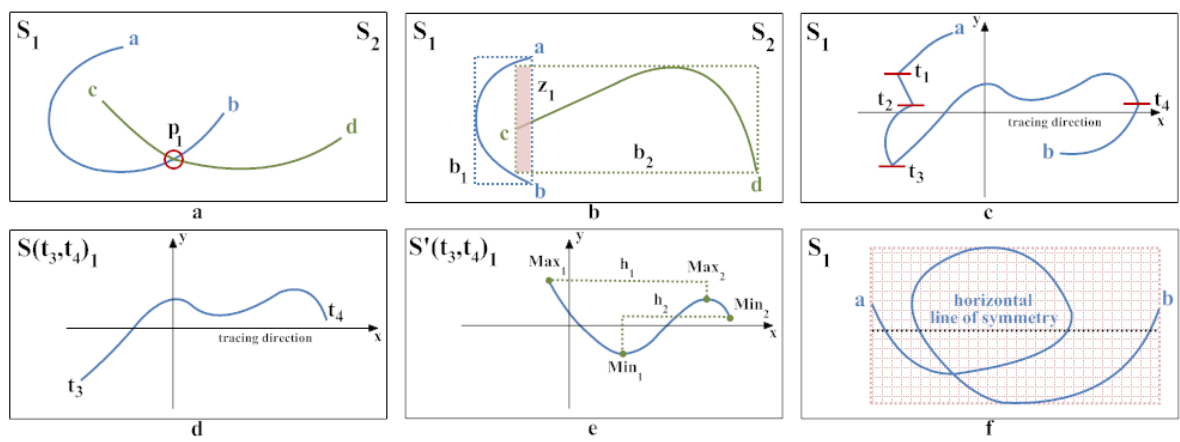

Fig. 2. ODE Feateurs: (a) Interception, (b) Closeness, (c)(d)(e) Curvature, (f) Entropy

partitioned sets of strokes (also isolated) representing the candidate objects. The interception feature (Fig. $2 \mathrm{a}$ ) is verified when two $\left(S_{1}\right.$ and $\left.S_{2}\right)$ or more strokes (not necessarily consecutive) have at least a common interception point $\left(p_{1}\right)$. Moreover if a set of sequential strokes are intercepted at the start and/or end points $(a, b, c$ and $d$ ) then they can be merged in a single stroke. The closeness feature (Fig. 2 $\mathrm{b}$ ) is verified when the bounding boxes $\left(b_{1}\right.$ and $\left.b_{2}\right)$ of two or more strokes (not necessarily consecutive) are overlapped ( $z_{1}$ zone) without having interception points: in this case sequential strokes must not be merged. The introduced features can support the multi-stroke drawing style as well as stroke structure simplification. Each stroke of each candidate object is further analyzed by curvature and entropy features to evaluate the homogeneity degree. The curvature feature measures the speed with which a stroke changes its trajectory. This task is achieved by dividing each stroke (when possible) in different sub-strokes (Fig. $2 \mathrm{k}:\left(a, t_{1}\right),\left(t_{1}, t_{2}\right),\left(t_{2}, t_{3}\right),\left(t_{3}, t_{4}\right)$, and $\left.\left(t_{4}, b\right)\right)$ according to the direction changes depending on the tracing direction (i.e., $\mathrm{x}$ axis), and by analyzing each of them (e.g., Fig. 2 d: $\left.\left(i_{3}, i_{4}\right)\right)$ by means of its derivative (Fig. 2 $\mathrm{e}$ ). The computation of the distances among maximum $\left(h_{1}\right)$ and minimum $\left(h_{2}\right)$ points of all derivatives can be considered a characterizing measure of the main stroke. The entropy feature measures the stochastic distribution of the pixels composing a stroke. This task is achieved by considering the pixel disposition above and below the horizontal line of symmetry of the bounding box containing the stroke (Fig. 2f) and comparing their messy level. Also in this case the computation of the absolute difference between the two sub-distributions can be considered a distinguishing measure of the stroke. In this phase, specific values of the introduced features are not required since we need to check the homogeneity of each candidate object (which is verified when all strokes belonging to it have similar/comparable values). If a candidate object is not homogeneous it is separated (when possible) by considering the interception feature more significant than the closeness feature. Subsequently on the new candidate objects the homogeneity degree is re-evaluated. The strategy can be re-applied until a set of strokes is obtained. The final result of the ODE process is to provide a set of 

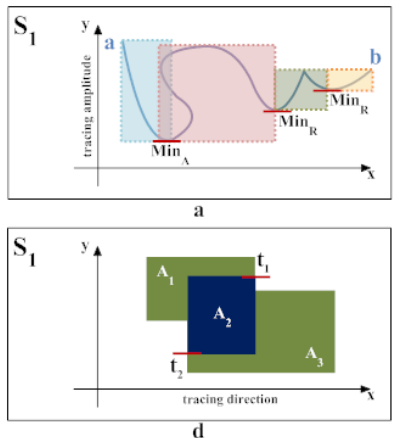
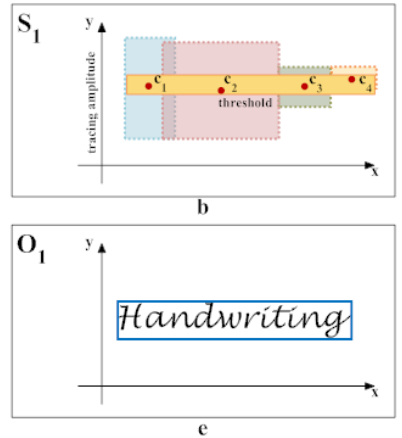
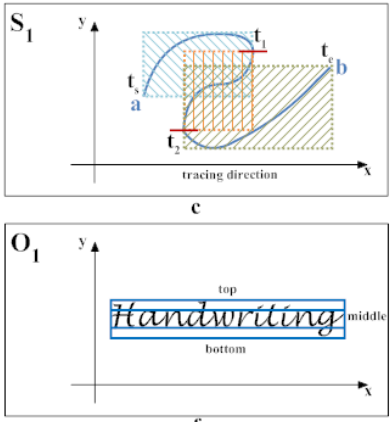

Fig. 3. DSE Feateurs: (a)(b) Linearity, (c)(d) Bounding Box Ratio, (e)(f) Band Ratio

homogeneous objects (also isolated strokes) whose strokes have well defined interception and/or closeness features with the others.

The DSE computes mathematical (i.e., linearity) and statistical (i.e., bounding box and band ratio) relationships on each candidate object to provide a reliable classification. The linearity feature measures the linearity of the stroke style. This task is achieved by building on each stroke (when possible) a set of bounding boxes (Fig. $3 \mathrm{a}$ ) in correspondence of the absolute and/or relative minimum points depending on the tracing amplitude (i.e., y axis), and measuring their linearity degree by the horizontal alignment of the related barycenters (Fig. 3b: $c_{1}, c_{2}, c_{3}$, and $c_{4}$ ) according to a parametric threshold depending on size and style of the stroke. A stroke without any minimum point is always considered linear. The bounding box ratio feature measures the density of the stroke style. This task is accomplished by considering again the bounding boxes related to the direction changes depending on the tracing direction (i.e., $\mathrm{x}$ axis) of the stroke (Fig. 3r), and by measuring the ratio (Fig. 3]d) between areas with $\left(A_{2}\right)$ and without $\left(A_{1}\right.$, and $\left.A_{3}\right)$ bounding box overlapping. The band ratio feature measures the distribution of the stroke style. This task is reached by computing on the bounding box containing the whole object (Fig. 3 3 : $\left.O_{1}\right)$ three bands (Fig. 3: top, middle, and bottom) representing a selective statistical distribution of the pixels composing the stroke. The middle band is built first than the others, and it is designed to maximize the quantity of pixels with respect of its area. In this phase, specific values of the introduced features have huge importance since they lead the distinguishing process. In particular, a candidate object containing a horizontal handwriting (uppercase and lowercase letters) is characterized by: high linearity, high bounding box ratio, high density of the middle band (or also equidistribution of the three bands). Similar considerations can be performed for vertical handwriting. Actually, the DSE process tries to identify each object as textual object; when it fails the object is identified as graphical object. The final task of the DSE is to route each object to the related recognizer. 


\subsection{Handwriting Recognizer Module}

Currently, there are a lot of on-line stroke-based OCR libraries and applications. We chosen to use Jarnal [16] for many reasons. First, it provided remarkable recognition rate. Second, it is cross-platform and open-source. Third, it is developed in Java (as our framework) and our stroke data structures are quite similar. Finally, it recognizes sentences composed of uppercase and lowercase letters.

\subsection{Freehand Drawing Recognizer Module}

This module is composed by five processes: First Layer Segmentation Algorithm (FLSA), Second Layer Segmentation Algorithm (SLSA), Constraint Determination Algorithm (CDA), Sketch Description Algorithm (SDA), and Matching Process (MP). The first and second transform any source freehand drawing in a set of default geometrical primitives and describe them in Scalable Vector Graphics (SVG) format. The third and fourth compute spatial constraints among primitives and describe them in XML format (merging these with the SVG information). Finally, the last performs the matching process between the vectorial representation of the graphical object (i.e., merged SVG/XML format) and the related representation of the library previously designed by a skilled user (i.e., user able to define a SVG/XML library following a simple protocol).

The FLSA provides two different set of shapes: closed regions and polylines. The first is defined (if any) as the smallest area contained by a set of strokes and/or sub-strokes. In this context, a sub-stroke is any part of any stroke included between two points, where each point can be of start, end or interception. The second is defined (if any) as the smallest not closed stroke or sub-stroke. Following these two definitions each freehand drawing can be decomposed into closed regions and/or polylines avoiding ambiguity issues. This task is accomplished in two steps. First, each stroke without interception points is checked to identify a closed region or an open polyline. Second, from each interception point of each stroke an exhaustive search process is carried out to detect the smallest area that does not overlap (entirely or in part) with other found areas. The process ends when all interception points are analyzed. The SLSA starts from closed regions and polylines, and provides their geometrical primitives: lines and ovals (including arcs and circles). The reason is that every stroke (or sub-stroke) can be approximated by them. The process starts by determining all lines of the shape, the rest of contiguous pixels are considered ovals. This task is performed in two steps. First, all longer sets of contiguous pixels having a linearity property are searched and classified as lines. In this context the linearity property indicates that linear contiguous pixels have to be contained within an oriented rectangle having a fixed minimum width and whose default height defines the admitted tolerance. Second, all discarded sets of contiguous pixels do not have linearity property since their oriented rectangles have a width lower than the fixed minimum width. All these sets are classified as ovals. Finally, the SLSA replaces pixels classified as lines and ovals with SVG line and SVG ellipse (part of oval with SVG shape), respectively. The CDA starts from SVG 

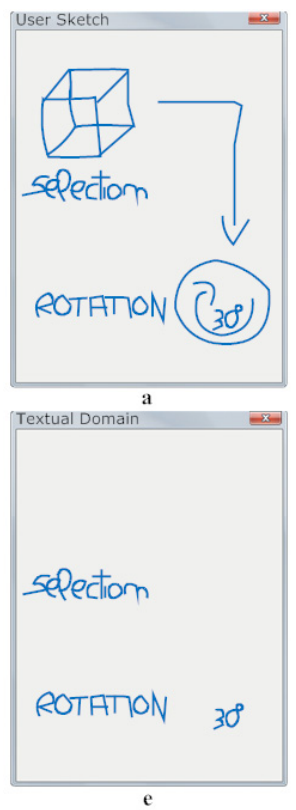
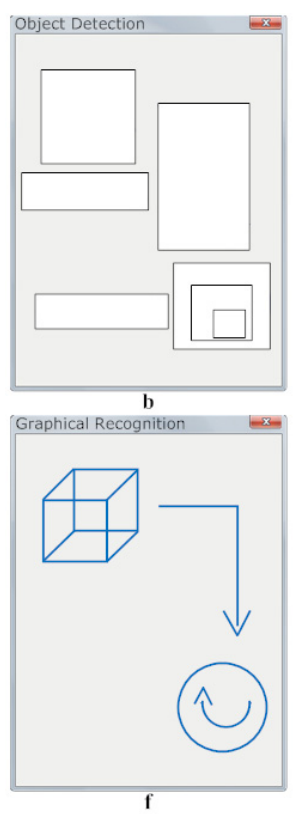
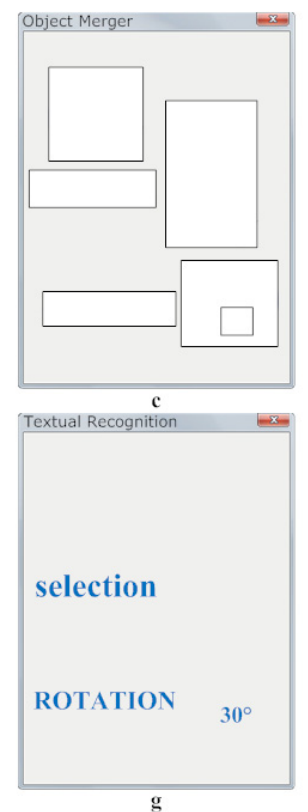
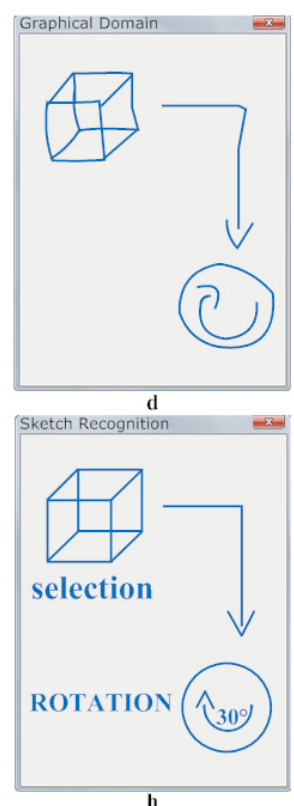

Fig. 4. Sketch Recognition: (a) User Sketch, (b) Seven Recognized Objects, (c) Merging Between Two Homogeneous Objects, (d) Graphical Domain, (e) Textual Domain, (f) Graphical Recognition, (g) Textual Recognition, (h) Sketch Recognition

primitives and detects the spatial relationships among them. This task is achieved by detecting on each SVG primitive (line, ellipse or path) of each shape the following constraints: intrinsics (e.g., orientation, closing), internals (e.g., coincident, intersect), and externals (e.g., contain, alignment). Each constraint has a set of exhaustive attributes to define every aspect of the spatial relationship (e.g., orientation degree, vertical or horizontal alignment). All these information are described in an XML format file. The SDA starts from the vectorial information of the SLSA (primitives expresses in SVG) and CDA (constraints expressed in XML) and provides a merged XML-like format file called Sketch Meta Language (SketchML) 6]. This versatile representation allows us to integrate the framework with compatibles services and/or applications. The MP compares each sketched freehand drawing with the vectorial ones contained within the library to identify the correct symbol (i.e., user action), and to replace it within the sketched layout (considering: size and orientation). Our framework allows skilled users to define any kind of library by using the Library Editor (LB) which defines graphical symbols through the mentioned SketckML. In fact, SketchML was designed to generate instances easily represented by graphs, where nodes and arcs symbolize primitives and constraints, respectively. The process performs an exhaustive comparison among each sketched symbol with those contained within the library. A rank strategy is adopted to choose the most suitable symbol. 


\section{Experimental Results}

We performed several experimental sessions to verify the reliability of the framework focusing on domain separation and freehand drawing recognition. Fig. 4 shows a concrete example of complex layout whose elements were separated and subsequently recognized. Experimental tests were performed on both homogeneous (textual or graphical objects) and heterogeneous (textual and graphical objects) layouts. In particular, 15 test users ( 7 right-handed and 2 left-handed men, 4 right-handed and 2 left-handed women) were employed to sketch 200 schemes (about 14 schemes per person) subdivided in 50 homogeneous (i.e., a single geometrical 2D or 3D shape, e.g., circle, square, cube, cone) and 150 heterogeneous (i.e., one or more shapes tagged with one or more textual labels composed by uppercase and/or lowercase letters). A heterogeneous layout was composed by no more than 5 different shapes and 5 different labels. In this direction, the example reported in Fig. 4 represents one of the more complex cases having 3 symbols and 3 labels. We acquired all sketches both by mouse and by tablet. Furthermore a set of selected homogeneous sketches (i.e., unistroke) were acquired by an RGB camera. In the first two cases the framework shown comparable behaviours obtaining remarkable results on separation (success rate: $85 \%$ ) and recognition (success rate: 94\%) tasks. An edit functionality of the sketch was implemented to allow users to modify (i.e., delete, redraw) the layout in case of separation failure. Also in the last case we obtained considerable results on recognition (success rate: 90\%) even though they were obtained by using a reduced library (only some well know $2 \mathrm{D}$ geometrical symbols). We also observed that the success rate could be influenced by the acquisition methods since the interpolation process to obtain the skeleton of the gesture is a real critical task. The framework includes also a supervised mechanism to overcome the recognition ambiguities on graphical symbols. When two or more symbols are recognized with similar ranks (distance in absolute value of about 10\%) they are presented to the user who can select the right one. Finally, the tests were performed on middle/low hardware level (Intel(R) Core(TM) i5-2430M CPU 2.40Ghz, RAM $4 \mathrm{~GB})$ obtaining separation and recognition results in real time.

\section{Conclusions}

Sketch-based interfaces allow users to convey concepts and/or commands using freehand drawing and/or handwriting. Domain separation and recognition algorithms, on the one hand, remain still fundamental issues of this kind of interfaces, on the other hand, can be suitably adjusted to support touch and touchless gesture-based interfaces. This paper presents SketchSPORE, a novel framework designed to distinguish and recognize elements of a sketch (in on-line and off-line modes) avoiding multi-domain and ambiguity issues. The framework processing is performed by SketchML to maintain a standard communication channel with service and/or application targets. Experimental tests allowed us to verify the method. Currently, we are working to improve library editor, separation and recognition rate, and matching process. 


\section{References}

1. Alvarado, C., Davis, R.: Sketchread: a multi-domain sketch recognition engine. In: ACM Symposium on User Interface Software and Technology, pp. 23-32 (2004)

2. Alvarado, C., Davis, R.: Dynamically constructed bayes nets for multi-domain sketch understanding. In: ACM SIGGRAPH 2007 Courses, SIGGRAPH 2007, pp. 1-6. ACM, New York (2007)

3. Avola, D., Bottoni, P., Dafinei, A., Labella, A.: Fcbd: An agent-based architecture to support sketch recognition interfaces. In: Proceedings of the 17th International Conference on Distributed Multimedia Systems, DMS 2011, pp. 295-300. Knowledge Systems Institute, Convitto della Calza (2011)

4. Avola, D., Del Buono, A., Gianforme, G., Paolozzi, S.: A novel recognition approach for sketch-based interfaces. In: Foggia, P., Sansone, C., Vento, M. (eds.) ICIAP 2009. LNCS, vol. 5716, pp. 1015-1024. Springer, Heidelberg (2009)

5. Avola, D., Del Buono, A., Del Nostro, P., Wang, R.: A novel online textual/graphical domain separation approach for sketch-based interfaces. In: Damiani, E., Jeong, J., Howlett, R.J., Jain, L.C. (eds.) New Directions in Intelligent Interactive Multimedia Systems and Services - 2. SCI, vol. 226, pp. 167-176. Springer, Heidelberg (2009)

6. Avola, D., Buono, A.D., Gianforme, G., Paolozzi, S., Wang, R.: Sketchml a representation language for novel sketch recognition approach. In: Proceedings of the 2nd International Conference on Pervasive Technologies Related to Assistive Environments, PETRA 2009, pp. 1-8. ACM, New York (2009)

7. Avola, D., Cinque, L., Placidi, G.: A novel multimodal framework to support advanced human-computer interaction. In: Proceedings of the 6th Group of Italian Researchers in Pattern Recognition, GIRPR 2012, pp. 1-12. GIRPR Press, Certosa di Pontignano (2012)

8. Bhat, A., Hammond, T.: Using entropy to distinguish shape versus text in handdrawn diagrams. In: Proceedings of the 21st International Jont Conference on Artifical Intelligence, IJCAI 2009, pp. 1395-1400. Morgan K. Publishers Inc., USA (2009)

9. Bishop, C.M., Svensn, M.: Distinguishing text from graphics in on-line handwritten ink. In: Proceedings of the 9th International Workshop on Frontiers in Handwriting Recognition, IWFHR 2004, pp. 142-147. IEEE Computer Society, USA (2004)

10. Blagojevic, R., Plimmer, B., Grundy, J., Wang, Y.: Using data mining for digital ink recognition: Dividing text and shapes in sketched diagrams. Computers \& Graphics 35(5), 976-991 (2011)

11. Caren (2013), http://www.motekmedical.com/products/caren/

12. Fonseca, M., Pimentel, C., Jorge, J.: Cali: An online scribble recognizer for calligraphic interfaces. In: AAAI 2002 Spring Symposium, AAAI 2002, pp. 51-58 (2002)

13. Fonseca, M.J., Jorge, J.A.: Experimental evaluation of an on-line scribble recognizer. Pattern Recognition Letters 22(12), 1311-1319 (2001)

14. Hammond, T., Davis, R.: Ladder: A language to describe drawing, display, and editing in sketch recognition. In: Gottlob, G., Walsh, T. (eds.) Proceedings of the 8th Int. Joint Conf. on Art. Intel., IJCAI 2003, pp. 461-467. M. Kaufmann (2003)

15. Hammond, T., Davis, R.: Ladder, a sketching language for user interface developers. Comput. Graph. 29(4), 518-532 (2005)

16. Jarnal (2013), http://jarnal.wikispaces.com/

17. Plamondon, R., Srihari, S.N.: On-line and off-line handwriting recognition: A comprehensive survey. IEEE Transactions on Pattern Analysis and Machine Intelligence 22(1), 63-84 (2000) 